\title{
On new spectral multiplicities for ergodic maps
}

\author{
by
}

\author{
Alexandre I. Danilenko (Kharkov)
}

\begin{abstract}
It is shown that each subset of positive integers that contains 2 is realizable as the set of essential values of the multiplicity function for the Koopman operator of some weakly mixing transformation.
\end{abstract}

0. Introduction. Let $(X, \mathfrak{B}, \mu)$ be a standard non-atomic probability space. Given a $\mu$-preserving (invertible) transformation $T$, we denote by $U_{T}$ the corresponding Koopman operator in $L^{2}(X, \mu), U_{T} f:=f \circ T$. Let $\mathcal{M}(T)$ stand for the set of essential values of the spectral multiplicity function for the restriction of $U_{T}$ to the subspace of 0 -mean functions, $L_{0}^{2}(X, \mu):=$ $L^{2}(X, \mu) \ominus \mathbb{C}$. We call a subset $M$ of positive integers realizable if there is an ergodic transformation $T$ such that $M=\mathcal{M}(T)$. In the present paper we investigate a long-standing open problem in the spectral theory of dynamical systems that can be stated as follows:

- What subsets of $\{1,2, \ldots\}$ are realizable?

It is expected that all subsets are realizable. It has already been shown that all subsets containing 1 are realizable [KL] (reproved with a different argument in $\mathrm{Ag} 2$ ). See also earlier works [Os], [R1], [R2], G-L] on the subject. We note that the spectral multiplicities from those papers are realized on transformations that are compact group extensions of rank-one maps.

Less is known about realizability of subsets without 1 . Is $\{n\}$ realizable for $n>1$ ? This problem of Rokhlin was first solved for $n=2$ in Ag1 and [Ry1]. The transformations considered in those papers are Cartesian squares of rank-one maps. Other realizable sets came with $n$-fold Cartesian products and their natural factors: $\{n, n(n-1), \ldots, n$ ! $\}$ in [Ag1], $\{2,3, \ldots, n\}$ in $\mathrm{Ag} 4$, etc. It is worth noting that those works on Cartesian products were influenced by the paper [Ka] which circulated since mid-eighties as an unpublished manuscript. As was shown in [Ry2], [Ry3] and [Ag4, those sets without 1 are also realizable in the class of mixing transformations. 
For an arbitrary $n$, the Rokhlin problem on homogeneous spectrum was first solved in Ag3 in a non-constructive way. An explicit solution appeared in [Da2]. A method of auxiliary non-Abelian group actions was in use in those two papers. The explicit construction from [Da2] combined with the techniques of compact group extensions was used to show that for each $n>1$ and a subset $M \subset \mathbb{N}$, the set $n \cdot(M \cup\{1\})$ is realizable [Da2].

Let $G$ be a countable Abelian group, $H$ a subgroup of $G$ and $v: G \rightarrow G$ a group automorphism. We set

$$
L(G, H, v):=\left\{\#\left(\left\{v^{i}(h) \mid i \in \mathbb{Z}\right\} \cap H\right) \mid h \in H \backslash\{0\}\right\} .
$$

It was shown in a recent paper $\mathrm{KaL}$ that $\{2\} \cup L(G, H, v)$ is realizable whenever $v$ is periodic. In particular, all subsets $\{2\} \cup n \cdot(M \cup\{1\})$, where $n>1$ and $M$ is a finite subset of $\mathbb{N}$, are realizable. That answers a question from [Ry3 (see also [Da3, Section 5]). However, $\mathrm{KaL}$ does not contribute to realization of infinite subsets because periodicity of $v$ bounds $L(G, H, v)$ to be finite. Moreover, it remains unclear whether every finite subset of $\mathbb{N}$ equals $L(G, H, v)$ for some triplet $(G, H, v)$ with $v$ periodic $\left({ }^{1}\right)$.

The purpose of the present paper is to prove the following theorem which extends the main result of $\mathrm{KaL}]$ to all subsets containing 2 .

MaIn TheOREm. Let $E$ be an arbitrary subset of positive integers. Then there is a weakly mixing transformation $S$ such that $\mathcal{M}(S)=E \cup\{2\}$.

Our method further develops the approach of $\mathrm{KaL}$. It is based upon the solution of the Rokhlin problem for $n=2$ Ag1, Ry1 and "symmetries" of some special compact group extensions. We make use of the $(C, F)$ construction (see the survey [Da3]) as a convenient tool to build dynamical systems and their extensions that have a prescribed "list" of weak limits for powers of Koopman operators restricted to some "components", i.e. invariant subspaces. This implies that two components are either unitarily equivalent or spectrally disjoint. It remains to count the number of components in every unitary equivalence class. Notice that Katok and Lemańczyk $\mathrm{KaL}$ study so-called double (non-Abelian) compact $K \rtimes_{v}(\mathbb{Z} / n \mathbb{Z})$-extensions of rank-one maps. Every such extension can be considered as a $K$-extension of a $\mathbb{Z} / n \mathbb{Z}$-extension; that is what "double" means here. A benefit of a double extension is that an important cohomology equation on the $K$-valued cocycle (see (2-1) below) holds automatically. In this paper we consider only single Abelian $K$-extensions. The equation (2-1) is satisfied due to a special choice of the cocycle. An advantage of our approach is that the automorphism $v$ entering into the equation need not be periodic. This leads to realizability of infinite subsets.

$\left({ }^{1}\right)$ The affirmative answer is given in my recent work "New spectral multiplicities for mixing transformations", arXiv:0908.1640. 
1. Algebraic lemma. The following algebraic statement is a key ingredient in the proof of the Main Theorem.

Algebraic Lemma. Given any subset $E \subset \mathbb{N}$, there exist a countable Abelian group $G$, a subgroup $H \subset G$ and an automorphism $v: G \rightarrow G$ such that $E=L(G, H, v)$. Moreover, the following properties are satisfied:

(i) the subgroup $\mathcal{G}:=\left\{a \in \widehat{G} \mid a \circ v^{m_{a}}=a\right.$ for some $\left.m_{a}>0\right\}$ is locally finite, countable and dense in $\widehat{G}$,

(ii) if $g_{1}, g_{2} \in G$ and $v^{i}\left(g_{1}\right) \neq g_{2}$ for all $i \in \mathbb{Z}$ then there is $a \in \mathcal{G}$ such that $\sum_{i=0}^{m_{a}-1} a\left(v^{i}\left(g_{1}\right)\right) \neq \sum_{i=0}^{m_{a}-1} a\left(v^{i}\left(g_{2}\right)\right)$,

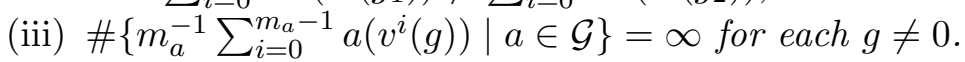

Proof. Since the case $E=\{1\}$ is trivial, we assume that $E \neq\{1\}$. Let $n_{1}, n_{2}, \ldots$ be a sequence of integers such that $E=\left\{n_{1}, n_{2}, \ldots,\right\}$ and $n_{1} \neq 1$. It is important that the sequence is infinite even if $E$ is finite (repetitions are allowed). We now set $G:=\bigoplus_{-\infty}^{+\infty} \mathbb{Z} / 2 \mathbb{Z}$. Let $v$ stand for the shift on $G$, i.e. if $g=\left(g_{i}\right)_{i \in \mathbb{Z}}$ then $(v(g))_{i}:=g_{i+1}$. To define $H$ we will first construct a sequence of finite subsets $A_{i} \subset \mathbb{Z}$ such that $\# A_{i} \rightarrow \infty$ and

$$
2 \max A_{i}<\min A_{i+1}
$$

for all $i=1,2, \ldots$. We then let $A:=\bigsqcup_{i=1}^{\infty} A_{i}$ and set $H:=\left\{\left(g_{i}\right)_{i=1}^{\infty} \in G \mid g_{i}=0\right.$ if $i \notin A$ and $g_{j}=g_{k}$ whenever $j, k \in A_{i}$ for some $\left.i\right\}$. The subsets $A_{i}$ are defined via an inductive procedure. In Step $k$ we define the subsets of cardinality $k$.

STEP 1. $A_{1}=1, A_{2}:=\{3\}, \ldots, A_{n_{1}}:=\left\{3^{n_{1}-1}\right\}$.

SteP $k+1$. Suppose that after Step $k$ we have already defined subsets $A_{1}, \ldots, A_{l_{k}}$. We call a subset $B \subset \bigsqcup_{i=1}^{l_{k}} A_{i}(k+1)$-basic if $\# B=k+1$ and if $A_{i} \cap B \neq \emptyset$ for some $1 \leq i \leq n_{k}$ implies $A_{i} \subset B$. Enumerate all the $(k+1)$-basic subsets as $B_{1}, \ldots, B_{r_{k}}$. Now put

$$
A_{l_{k}+r n_{k+1}+s}:=B_{r+1}+i_{r n_{k+1}+s} \quad \text { for all } 0 \leq r<r_{k}, 1 \leq s<n_{k+1},
$$

where the positive integers $\left(i_{j}\right)_{1 \leq j \leq\left(n_{k+1}-1\right) r_{k}}$ are chosen so that (1-1) is satisfied.

We now verify the conclusion of the lemma for the triplet $(G, H, v)$. Take $g=\left(g_{i}\right)_{i \in \mathbb{Z}} \in H$. Let $C:=\left\{i \mid g_{i} \neq 0\right\}$. Notice that $g_{i}=g_{j}=1$ for all $i, j \in C$. Denote by $p$ the cardinality of $C$. Then $p$ is the smallest number such that $C \subset \bigsqcup_{i \leq l_{p}} A_{i}$. By construction, there is a $k$-basic subset $B$ such that $C$ is a translation of $B$ in $\mathbb{Z}$. Moreover, there exist exactly $n_{p}$ different translations of $C$ which are inside $A$. This means that the $v$-orbit of $g$ intersects $H$ exactly $n_{p}$ times. Therefore $L(G, H, v) \subset E$. The converse inclusion is obvious. Thus the first claim of the lemma is shown.

It is easy to see that (i) is satisfied. 
Let $\mathcal{G}_{m}:=\left\{a \in \mathcal{G} \mid \widehat{v}^{m}(a)=a\right\}$. Then $\mathcal{G}_{m}$ is a finite $\widehat{v}$-invariant subgroup of $\mathcal{G}$. If $g_{1}, g_{2} \in G$ and $v^{i}\left(g_{1}\right) \neq g_{2}$ for all $i \in \mathbb{Z}$ then we can find $m>0$ such that $v^{i}\left(g_{1}\right)\left\lceil\mathcal{G}_{m} \neq g_{2}\left\lceil\mathcal{G}_{m}\right.\right.$ for all $i \in \mathbb{Z}$. Then

$$
\left(\sum _ { i = 0 } ^ { m } v ^ { i } ( g _ { 1 } ) \lceil \mathcal { G } _ { m } ) \perp \left(\sum_{i=0}^{m} v^{i}\left(g_{2}\right)\left\lceil\mathcal{G}_{m}\right) \quad \text { as elements of } L^{2}\left(\mathcal{G}_{m}\right),\right.\right.
$$

and (ii) follows.

We consider an element $0 \neq g \in G$ as an infinite sequence of symbols 0 and 1 with finitely many, say $j$, symbols 1 . Take a block $b \in(\mathbb{Z} / 2 \mathbb{Z})^{m}$ consisting of one symbol 1 and $m-1$ symbols 0 for a very large $m$. Then we set $a:=b^{\infty} \in \mathcal{G}_{m}$. It is easy to verify that $m^{-1} \sum_{i=0}^{m-1} a\left(v^{i}(g)\right)=(m-2 j) / m$. This yields (iii).

Notice that stronger versions of the above result have been established in some particular cases:

(o) If $1 \in E$ then $v$ in the statement of the Algebraic Lemma can be chosen quasi-periodic, i.e. every $v$-orbit is finite. If, in addition, $E$ is finite then $E=L(G, H, v)$ for finite-dimensional tori $G$ and $H$ and a periodic automorphism $v$ KL].

(o) If $E=\{2\} \cup n \cdot(M \cup\{1\})$, where $n>1$ and $M$ is a finite subset of $\mathbb{N}$, then $E=L(G, H, v)$ for finite groups $G$ and $H$ and a periodic automorphism $v[\mathrm{KaL}$.

In this connection we note that $v$ in our construction is not quasi-periodic even for $E$ finite or $1 \in E$.

2. Weak limits of powers, cocycles, and $(C, F)$-construction. We will need two lemmata on spectral properties of some Cartesian products. For the proof we refer to $\mathrm{Ag} 1, \mathrm{Ry} 1$ and $\mathrm{KaL}$.

LEMma 2.1 ( Ag1, Ry1). Let $T$ be a weakly mixing transformation with simple spectrum. If the weak closure of the powers of $U_{T}$ contains $0.5\left(I+U_{T}\right)$ then $T \times T$ has a homogeneous spectrum of multiplicity 2 in the orthocomplement to the constants.

We note that a theory of linked approximation suggested in [KaS] plays an important role in the proof of the above lemma.

Lemma $2.2([\mathrm{KaL}])$. Let $V_{i}, i=1,2$, be unitary operators with simple spectrum. Assume moreover that there are two sequences $\left(n_{t}\right)_{t>0}$ and $\left(m_{t}\right)_{t>0}$ such that

(i) $V_{i}^{n_{t}} \rightarrow 0.5\left(I+V_{i}^{*}\right)$ weakly, $i=1,2$.

(ii) $V_{i}^{m_{t}} \rightarrow 0.5\left(I+c_{i} V_{i}^{*}\right)$ weakly, $i=1,2$.

If $c_{1} \neq c_{2}$ then $V_{1} \otimes V_{2}$ also has a simple spectrum. 
Let $T$ be an ergodic transformation of $(X, \mu)$. Denote by $\mathcal{R} \subset X \times X$ the $T$-orbit equivalence relation. A Borel map $\alpha$ from $\mathcal{R}$ to a compact group $K$ is called a cocycle of $\mathcal{R}$ if

$$
\alpha(x, y) \alpha(y, z)=\alpha(x, z) \quad \text { for all }(x, y),(y, z) \in \mathcal{R} .
$$

Two cocycles $\alpha, \beta: \mathcal{R} \rightarrow K$ are cohomologous if

$$
\alpha(x, y)=\phi(x) \beta(x, y) \phi(y)^{-1} \text { at a.a. }(x, y) \in \mathcal{R}
$$

for a Borel map $\phi: X \rightarrow K$. If a transformation $S$ commutes with $T$ (i.e. $S \in C(T)$ ) then a cocycle $\alpha \circ S: \mathcal{R} \rightarrow K$ is well defined by $\alpha \circ S(x, y):=$ $\alpha(S x, S y)$. The important cohomology equation on $\alpha$ mentioned in Section 0 can now be stated as follows:

$$
\alpha \circ S \text { is cohomologous to } v \circ \alpha
$$

for some $S \in C(T)$ and a group automorphism $v: K \rightarrow K$.

To prove the Main Theorem we will use the $(C, F)$-construction (see [Ju], Da1]-Da3]). We now briefly outline its formalism. Let two sequences $\left(C_{n}\right)_{n>0}$ and $\left(F_{n}\right)_{n \geq 0}$ of finite subsets in $\mathbb{Z}$ be given such that:

- $F_{n}=\left\{0,1, \ldots, h_{n}-1\right\}, h_{0}=1, \# C_{n}>1$,

- $F_{n}+C_{n+1} \subset F_{n+1}$,

- $\left(F_{n}+c\right) \cap\left(F_{n}+c^{\prime}\right)=\emptyset$ if $c \neq c^{\prime}, c, c^{\prime} \in C_{n+1}$,

- $\lim _{n \rightarrow \infty} \frac{h_{n}}{\# C_{1} \cdots \# C_{n}}<\infty$.

Let $X_{n}:=F_{n} \times C_{n+1} \times C_{n+2} \times \cdots$. Endow this set with the (compact Polish) product topology. The map

$$
\left(f_{n}, c_{n+1}, c_{n+2}\right) \mapsto\left(f_{n}+c_{n+1}, c_{n+2}, \ldots\right)
$$

is a topological embedding of $X_{n}$ into $X_{n+1}$. We now set $X:=\bigcup_{n \geq 0} X_{n}$ and endow it with the (locally compact Polish) inductive limit topology. Given $A \subset F_{n}$, we denote by $[A]_{n}$ the cylinder $\left\{x=\left(f, c_{n+1}, \ldots\right) \in X_{n} \mid f \in A\right\}$. Then $\left\{[A]_{n} \mid A \subset F_{n}, n>0\right\}$ is the family of all compact open subsets in $X$. It forms a base of the topology on $X$.

Let $\mathcal{R}$ stand for the tail equivalence relation on $X$ : two points $x, x^{\prime} \in X$ are $\mathcal{R}$-equivalent if there is $n>0$ such that $x=\left(f_{n}, c_{n+1}, \ldots\right), x^{\prime}=$ $\left(f_{n}^{\prime}, c_{n+1}^{\prime}, \ldots\right) \in X_{n}$ and $c_{m}=c_{m}^{\prime}$ for all $m>n$. There is only one probability (non-atomic) Borel measure $\mu$ on $X$ which is invariant (and ergodic) under $\mathcal{R}$.

Now we define a transformation $T$ of $(X, \mu)$ by setting

$$
T\left(f_{n}, c_{n+1}, \ldots\right):=\left(1+f_{n}, c_{n+1}, \ldots\right) \text { whenever } f_{n}<h_{n}-1, n>0 .
$$

This formula defines $T$ partly on $X_{n}$. When $n \rightarrow \infty, T$ extends to the entire $X$ minus countably many points as a $\mu$-preserving transformation. 
Moreover, the $T$-orbit equivalence relation coincides with $\mathcal{R}$ (on the subset where $T$ is defined). We call $T$ the $(C, F)$-transformation associated with $\left(C_{n+1}, F_{n}\right)_{n \geq 0}$.

We recall the concept of $(C, F)$-cocycle (see [Da2]). From now on, the group $K$ is assumed Abelian. Given a sequence of maps $\alpha_{n}: C_{n} \rightarrow K$, $n=1,2, \ldots$, we first define a Borel cocycle $\alpha: \mathcal{R} \cap\left(X_{0} \times X_{0}\right) \rightarrow K$ by setting

$$
\alpha\left(x, x^{\prime}\right):=\sum_{n>0}\left(\alpha_{n}\left(c_{n}\right)-\alpha_{n}\left(c_{n}^{\prime}\right)\right)
$$

whenever $x=\left(0, c_{1}, c_{2}, \ldots\right) \in X_{0}, x^{\prime}=\left(0, c_{1}^{\prime}, c_{2}^{\prime}, \ldots\right) \in X_{0}$ and $\left(x, x^{\prime}\right) \in \mathcal{R}$. To extend $\alpha$ to the entire $\mathcal{R}$, we first define a map $\pi: X \rightarrow X_{0}$ as follows. Given $x \in X$, let $n$ be the least positive integer such that $x \in X_{n}$. Then $x=\left(f_{n}, c_{n+1}, \ldots\right) \in X_{n}$. We set

$$
\pi(x):=(\underbrace{0, \ldots, 0}_{n+1 \text { times }}, c_{n+1}, c_{n+2}, \ldots) \in X_{0} .
$$

Of course, $(x, \pi(x)) \in \mathcal{R}$. Now for each pair $(x, y) \in \mathcal{R}$, we let

$$
\alpha(x, y):=\alpha(\pi(x), \pi(y)) .
$$

It is easy to verify that $\alpha$ is a well defined cocycle of $\mathcal{R}$ with values in $K$. We call it the $(C, F)$-cocycle associated with $\left(\alpha_{n}\right)_{n=1}^{\infty}$.

The following statement follows from [Da2, Section 4].

Lemma 2.3. Let $\bar{z}=\left(z_{n}\right)_{n=1}^{\infty}$ be a sequence of positive reals. Suppose that

$$
\sum_{n>0} \#\left(C_{n} \triangle\left(C_{n}-z_{n}\right)\right) / \# C_{n}<\infty .
$$

For each $m>0$, set

$$
X_{m}^{\bar{z}}:=\left\{0,1, \ldots, h_{m}-z_{1}-\cdots-z_{m}\right\} \times \prod_{n>m}\left(C_{n} \cap\left(C_{n}-z_{n}\right)\right) \subset X_{m} .
$$

Then a transformation $S_{\bar{z}}$ of $(X, \mu)$ is well defined by setting

$$
S_{\bar{z}}(x):=\left(z_{1}+\cdots+z_{m}+f_{m}, z_{m+1}+c_{m+1}, z_{m+2}+c_{m+2}, \ldots\right)
$$

for all $x=\left(f_{m}, c_{m+1}, c_{m+2}, \ldots\right) \in X_{m}^{\bar{z}}, m=1,2, \ldots$ Moreover, $S_{\bar{z}}$ commutes with $T$, and $T^{z_{1}+\cdots+z_{m}} \rightarrow S_{\bar{z}}$ as $m \rightarrow \infty$.

Now let $C_{m}^{\circ}:=\left\{c \in C_{m} \cap\left(C_{m}-z_{m}\right) \mid \alpha_{m}\left(c+z_{m}\right)=v\left(\alpha_{m}(c)\right)\right\}$. If

$$
\sum_{n>0}\left(1-\# C_{n}^{\circ} / \# C_{n}\right)<\infty
$$

then the cocycle $\alpha \circ S_{\bar{z}}$ is cohomologous to $v \circ \alpha$. 
3. Proof of the Main Theorem. By the Algebraic Lemma, there exist a compact Polish Abelian group $K$, a closed subgroup $H$ of $K$ and a continuous automorphism $v$ of $K$ such that

$$
E=L(\widehat{K}, \widehat{K / H}, \widehat{v})
$$

We also assume that conditions (i)-(iii) from the statement of the lemma are satisfied. The subgroup of $v$-periodic points in $K$ will be denoted by $\mathcal{K}$.

We will construct some special $(C, F)$-transformation and its cocycle with values in $K$. Fix a partition

$$
\mathbb{N}=\bigsqcup_{a \in \mathcal{K}} \mathcal{N}_{a} \sqcup \bigsqcup_{a, b \in \mathcal{K}} \mathcal{N}_{a, b}
$$

of $\mathbb{N}$ into infinite subsets. Now we define a sequence $\left(C_{n}, h_{n}, z_{n}, \alpha_{n}\right)_{n=1}^{\infty}$ via an inductive procedure. Suppose we have already constructed this sequence up to index $n$. Consider now two cases.

[I] If $n+1 \in \mathcal{N}_{a}$ for some $a \in \mathcal{K}$, we denote by $m_{a}$ the least positive period of $a$ under $v$. Now we set

$$
\begin{gathered}
z_{n+1}:=m_{a} n h_{n}, \quad r_{n}:=n^{3} m_{a}, \\
C_{n+1}:=h_{n} \cdot\left\{0,1, \ldots, r_{n}-1\right\}, \quad h_{n+1}:=r_{n} h_{n},
\end{gathered}
$$

Let $\alpha_{n+1}: C_{n+1} \rightarrow K$ be any map satisfying the following conditions:

(A1) $\alpha_{n+1}\left(c+z_{n+1}\right)=v \circ \alpha_{n+1}(c)$ for all $c \in C_{n+1} \cap\left(C_{n+1}-z_{n+1}\right)$,

(A2) for each $0 \leq i<m_{a}$ there is a subset $C_{n+1, i} \subset C_{n+1}$ such that

$$
\begin{gathered}
C_{n+1, i}-h_{n} \subset C_{n+1}, \\
\alpha_{n+1}(c)=\alpha_{n+1}\left(c-h_{n}\right)+v^{i}(a) \quad \text { for all } c \in C_{n+1, i}, \\
\left|\frac{\# C_{n+1, i}}{\# C_{n+1}}-\frac{1}{m_{a}}\right|<\frac{2}{n m_{a}} .
\end{gathered}
$$

[II] If $n+1 \in \mathcal{N}_{a, b}$ for some $a, b \in \mathcal{K}$, we denote by $m_{a, b}$ the least common positive period of $a$ and $b$ under $v$. Now we set

$$
\begin{gathered}
z_{n+1}:=m_{a, b} n\left(2 h_{n}+1\right), \quad r_{n}:=2 n^{3} m_{a, b}, \\
D_{n+1}:=h_{n} \cdot\left\{0,1, \ldots, n m_{a, b}-1\right\} \sqcup\left(\left(h_{n}+1\right) \cdot\left\{1, \ldots, n m_{a, b}\right\}+h_{n}\left(n m_{a, b}-1\right)\right), \\
C_{n+1}:=D_{n+1}+z_{n+1} \cdot\left\{0,1, \ldots, n^{2}-1\right\}, \quad h_{n+1}:=r_{n} h_{n}+r_{n} / 2 .
\end{gathered}
$$

Let $\alpha_{n+1}: C_{n+1} \rightarrow K$ be any map satisfying the following conditions:

(A3) $\alpha_{n+1}\left(c+z_{n+1}\right)=v \circ \alpha_{n+1}(c)$ for all $c \in C_{n+1} \cap\left(C_{n+1}-z_{n+1}\right)$,

(A4) for each $e \in\{a, b\}$ and $0 \leq i<m_{a}$ there is a subset $C_{n+1, i}^{e} \subset C_{n+1}$ such that 


$$
\begin{gathered}
C_{n+1, i}^{e}-h_{n}-1 \subset C_{n+1}, \\
\alpha_{n+1}(c)=\alpha_{n+1}\left(c-h_{n}-1\right)+v^{i}(e) \quad \text { for all } c \in C_{n+1, i}^{e}, \\
\left|\frac{\# C_{n+1, i}^{e}}{\# C_{n+1}}-\frac{1}{2 m_{a, b}}\right|<\frac{2}{n m_{a, b}} .
\end{gathered}
$$

Thus, $C_{n+1}, h_{n+1}, z_{n+1}, \alpha_{n+1}$ are completely defined.

We now let $F_{n}:=\left\{0,1, \ldots, h_{n}-1\right\}$. Denote by $(X, \mu, T)$ the $(C, F)$ transformation associated with the sequence $\left(C_{n+1}, F_{n}\right)_{n \geq 0}$. Let $\mathcal{R}$ stand for the tail equivalence relation (or, equivalently, $T$-orbit equivalence relation) on $X$. Denote by $\alpha: \mathcal{R} \rightarrow K$ the cocycle of $\mathcal{R}$ associated with the sequence $\left(\alpha_{n}\right)_{n>0}$. Let $\lambda_{K / H}$ stand for the Haar measure on $K / H$. We denote by $T_{\alpha, H}$ the following transformation of the space $\left(X \times K / H, \lambda_{K / H}\right)$ :

$$
T_{\alpha, H}(x, k+H):=(T x, \alpha(T x, x)+k+H) .
$$

Our purpose is to prove that $\mathcal{M}\left(T \times T_{\alpha, H}\right)=E \cup\{2\}$.

Since

$$
\sum_{n>0} \frac{\#\left(C_{n} \triangle\left(C_{n}-z_{n}\right)\right)}{\# C_{n}}=\sum_{n>0} \frac{2}{n^{2}},
$$

it follows from Lemma 2.3 that a transformation $S_{\bar{z}}$ of $(X, \mu)$ is well defined by the formula $(2-2)$ and $S_{\bar{z}} \in C(T)$.

It follows from (A1) and (A3) that (2-3) is satisfied. Hence by Lemma 2.3, the cocycle $\alpha \circ S_{\bar{z}}$ is cohomologous to $v \circ \alpha$.

We need more notation. Given $a \in \mathcal{K}$ and $\chi \in \widehat{K}$, let

$$
l_{\chi}(a):=m_{a}^{-1} \sum_{i=0}^{m_{a}-1} \chi\left(v^{i}(a)\right) .
$$

We also denote by $U_{T, \chi}$ the unitary operator on the space $L^{2}(X, \mu)$ given by

$$
U_{T, \chi} g(x)=\chi(\alpha(T x, x)) g(T x) .
$$

Lemma 3.1. Let $a, b \in \mathcal{K}$. Then for each $\chi \in \widehat{K}$,

(i) $U_{T, \chi}^{h_{n}} \rightarrow l_{\chi}(a) \cdot I$ as $\mathcal{N}_{a}-1 \ni n \rightarrow \infty$,

(ii) $U_{T, \chi}^{h_{n}} \rightarrow 0.5\left(l_{\chi}(a) \cdot I+l_{\chi}(b) \cdot U_{T, \chi}^{*}\right)$ as $\mathcal{N}_{a, b}-1 \ni n \rightarrow \infty$.

Proof. We show only (ii) since (i) is proved in a similar way but a bit simpler. Let $n \in \mathcal{N}_{a, b}$.

Take any subset $A \subset F_{n}$. We note that $[A]_{n}=\left[A+C_{n+1}\right]_{n+1}$. Therefore it follows from (A4) that for each $x \in T\left[F_{n}\right]_{n}$, 


$$
\begin{aligned}
U_{T, \chi}^{h_{n}} 1_{[A]_{n}}(x)= & \sum_{e \in\{a, b\}} \sum_{i=0}^{m_{a, b}-1} \chi\left(\alpha\left(T^{h_{n}} x, x\right)\right) 1_{\left[A+C_{n+1, i}^{e}\right]_{n+1}}\left(T^{h_{n}} x\right)+h(x) \\
= & \sum_{i=0}^{m_{a, b}-1} \chi\left(v^{i}(a)\right) 1_{\left[A+C_{n+1, i}^{a}-h_{n}\right]_{n+1}}(x) \\
& +\sum_{i=0}^{m_{a, b}-1} \chi\left(v^{i}(b) \alpha\left(T^{-1} x, x\right)\right) 1_{\left[A+C_{n+1, i}^{b}-h_{n}-1\right]_{n+1}}\left(T^{-1} x\right)+h(x) \\
= & \sum_{i=0}^{m_{a, b}-1} \chi\left(v^{i}(a)\right) 1_{\left[A+C_{n+1, i}^{a}-h_{n}\right]_{n+1}}(x) \\
& +\sum_{i=0}^{m_{a, b}-1} \chi\left(v^{i}(b)\right) U_{T, \chi}^{*} 1_{\left[A+C_{n+1, i}^{b}-h_{n}-1\right]_{n+1}}(x)+h(x),
\end{aligned}
$$

where $x \mapsto h(x)$ is a function whose $L^{2}$-norm is small. Hence

$$
U_{T, \chi}^{h_{n}}-\sum_{i=0}^{m_{a, b}-1} \chi\left(v^{i}(a)\right) 1_{\left[C_{n+1, i}^{a}-h_{n}\right]_{n+1}}-\sum_{i=0}^{m_{a, b}-1} \chi\left(v^{i}(b)\right) U_{T, \chi}^{*} 1_{\left[C_{n+1, i}^{b}-h_{n}-1\right]_{n+1}} \rightarrow 0
$$

weakly as $\mathcal{N}_{a, b}-1 \ni n \rightarrow \infty$, where the functions

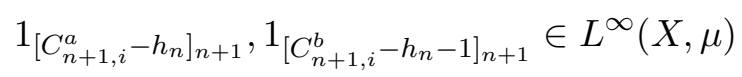

are considered as multiplication operators in $L^{2}(X, \mu)$.

It remains to use the inequalities from (A2) and (A4) and a standard fact that for any sequence $C_{n}^{\prime} \subset C_{n}$ such that $\# C_{n}^{\prime} / \# C_{n} \rightarrow \delta$ for some $\delta>0$ we have

$$
1_{\left[C_{n}^{\prime}\right]_{n}} \rightarrow \delta I \quad \text { weakly as } n \rightarrow \infty \text {. }
$$

Proof of Main Theorem. Lemma 3.1 implies that the transformation $T_{\alpha}$ (and hence its factor $T_{\alpha, H}$ ) is weakly mixing.

To show that $\mathcal{M}\left(T \times T_{\alpha, H}\right)=E \cup\{2\}$ we consider a natural decomposition of $U_{T \times T_{\alpha, H}}$ into an orthogonal sum

$$
U_{T \times T_{\alpha, H}}=\bigoplus_{\chi \in \widehat{K / H}}\left(U_{T} \otimes U_{T, \chi}\right) .
$$

It is enough to prove the following:

(a) $U_{T} \otimes U_{T}$ has homogeneous spectrum of multiplicity 2 in the orthocomplement to the constants,

(b) $U_{T} \otimes U_{T, \chi}$ has simple spectrum if $\chi \neq 0$,

(c) $U_{T} \otimes U_{T, \chi}$ and $U_{T} \otimes U_{T, \xi}$ are unitarily equivalent if $\chi$ and $\xi$ belong to the same $\widehat{v}$-orbit, 
(d) the measures of maximal spectral type of $U_{T} \otimes U_{T, \chi}$ and $U_{T} \otimes U_{T, \xi}$ are mutually singular if $\chi$ and $\xi$ are not on the same $\widehat{v}$-orbit.

By Lemma 3.1(ii), $U_{T}^{h_{n}} \rightarrow 0.5\left(I+U_{T}^{*}\right)$ as $\mathcal{N}_{0,0}-1 \ni n \rightarrow \infty$. Therefore (a) follows from Lemma 2.1.

Since $T$ is of rank one and the map $[f] \ni x \mapsto \alpha(T x, x) \in K$ is constant for each $f \in F_{n} \backslash\left\{h_{n}-1\right\}, n \in \mathbb{N}$, it follows that the operator $U_{T, \chi}$ has simple spectrum. Moreover, $U_{T, \chi}^{h_{n}} \rightarrow 0.5\left(I+U_{T, \chi}^{*}\right)$ as $\mathcal{N}_{0,0}-1 \ni n \rightarrow \infty$ by Lemma 3.1(ii). Since $\chi$ is nontrivial, it follows from claim (ii) of the Algebraic Lemma that there is $a \in \mathcal{K}$ with $l_{\chi}(a) \neq 1$. By Lemma 3.1(ii),

$$
U_{T}^{h_{n}} \rightarrow 0.5\left(I+U_{T}^{*}\right) \quad \text { but } \quad U_{T, \chi}^{h_{n}} \rightarrow 0.5\left(I+l_{\chi}(a) U_{T, \chi}^{*}\right)
$$

as $\mathcal{N}_{0, a}-1 \ni n \rightarrow \infty$. Therefore Lemma 2.2 implies (b).

Since the cocycles $\alpha \circ S_{\bar{z}}$ and $v \circ \alpha$ are cohomologous, $U_{T, \chi}$ and $U_{T, \xi}$ are unitarily equivalent whenever $\chi$ and $\xi$ lie on the same orbit of $\widehat{v}$ (see [G-L], [KL]). This yields (c).

To prove (d), we first find $a \in \mathcal{G}$ such that $l_{\chi}(a) \neq l_{\xi}(a)$ (see claim (ii) of the Algebraic Lemma). It follows from Lemma 3.1(i) that

$$
U_{T}^{h_{n}} \otimes U_{T, \chi}^{h_{n}} \rightarrow l_{\chi}(a) I \quad \text { and } \quad U_{T}^{h_{n}} \otimes U_{T, \xi}^{h_{n}} \rightarrow l_{\xi}(a) I
$$

as $\mathcal{N}_{a}-1 \ni n \rightarrow \infty$. Hence (d) holds.

4. Concluding remarks. Combining the techniques developed in $\mathrm{KaL}$ with our approach one can also realize some subsets of $\mathbb{N} \backslash\{1,2\}$. For instance, given any subset $E \subset \mathbb{N}$, let $T_{\alpha, H}$ denote the skew product transformation constructed in Section 3. Then

$$
\mathcal{M}\left(T^{\times k} \times T_{\alpha, H}\right)=\{k+1,(k+1) k, \ldots,(k+1) !\} \cup\{k, k(k-1), \ldots, k !\} \cdot E .
$$

For example, taking $k=2$ we obtain $\{3,6\} \cup 2 \cdot E$. To show (4-1) we repeat the proof of the Main Theorem almost literally but apply the following lemma instead of Lemma 2.2.

Lemma 4.2 ([KaL, Proposition 1], [DaR, Lemma 1.2]). Let $V$ and $W$ be unitary operators with simple spectrum in Hilbert spaces $\mathcal{H}$ and $\widetilde{\mathcal{H}}$ respectively. Assume moreover that for each $i=1, \ldots, k$, there are two sequences $n_{t}^{(i)} \rightarrow \infty$ and $m_{t}^{(i)} \rightarrow \infty$ and complex numbers $\kappa_{i} \neq \widetilde{\kappa}_{i}$ such that

(i) $V^{n_{t}^{(i)}} \rightarrow 0.5\left(\kappa_{i} I+V^{*}\right), W^{n_{t}^{(i)}} \rightarrow 0.5\left(\kappa_{i} I+W^{*}\right)$ weakly,

(ii) $V^{m_{t}^{(i)}} \rightarrow 0.5\left(\kappa_{i} I+V_{i}^{*}\right), W^{m_{t}^{(i)}} \rightarrow 0.5\left(\widetilde{\kappa}_{i} I+W^{*}\right)$ weakly and $\#\left\{\kappa_{1}, \ldots, \kappa_{k}\right\}=k$. Then $V^{\odot k} \otimes W$ has a simple spectrum.

A more general class of multiplicities arises when considering natural factors of $T^{\times k} \times T_{\alpha, H}$ as in $\mathrm{Ag} 4$. Moreover, Ryzhikov recently constructed a new series of realizable sets that do not belong to this class Ry4. For 
instance, the sets $\{p, q, p q\}$ are realizable for all $p, q>0$. The sets $\{3,4\}$ and $\{3,5\}$ are the simplest ones that are covered neither by this class nor by Ryzhikov's.

We also note that while our approach based on the Algebraic Lemma and weak limits techniques is not sufficient to solve the spectral multiplicity problem completely in the framework of ergodic finite measure preserving transformations, it can be adapted to the framework of infinite measure preserving maps to show the following: every subset of $\mathbb{N}$ is realizable on an ergodic infinite measure preserving transformation. This is done in a forthcoming paper of Ryzhikov and the author [DaR].

It is also interesting to study the spectral multiplicity problem for general Abelian group actions. Some results in this field have recently been obtained by Konev, a post-graduate student of Ryzhikov, for $\mathbb{Z}^{2}$-actions [Ko].

\section{References}

[Ag1] O. N. Ageev, On ergodic transformations with homogeneous spectrum, J. Dynam. Control Systems 5 (1999), 149-152.

[Ag2] - On the multiplicity function of generic group extensions with continuous spectrum, Ergodic Theory Dynam. Systems 21 (2001), 321-338.

[Ag3] —, The homogeneous spectrum problem in ergodic theory, Invent. Math. 160 (2005), 417-446.

[Ag4] -, Mixing with staircase multiplicity fuction, Ergodic Theory Dynam. Systems 28 (2008), 1687-1700.

[Da1] A. I. Danilenko, Funny rank one weak mixing for nonsingular Abelian actions, Israel J. Math. 121 (2001), 29-54.

[Da2] - Explicit solution of Rokhlin's problem on homogeneous spectrum and applications, Ergodic Theory Dynam. Systems 26 (2006), 1467-1490.

[Da3] - , $(C, F)$-actions in ergodic theory, in: Geometry and Dynamics of Groups and Spaces Progr. Math. 265 (2008), 325-351.

[DaR] A. I. Danilenko and V. V. Ryzhikov, Spectral multiplicities for infinite measure preserving transformations, Preprint, arXiv:0905.3486.

[G-L] G. R. Goodson, J. Kwiatkowski, M. Lemańczyk and P. Liardet, On the multiplicity function of ergodic group extensions of rotations, Studia Math. 102 (1992), 157174 .

[Ju] A. del Junco, A simple map with no prime factors, Israel J. Math. 104 (1998), 301-320.

[Ka] A. B. Katok, Combinatorial Constructions in Ergodic Theory and Dynamics, Univ. Lecture Ser. 30, Amer. Math. Soc., Providence, RI, 2003.

[KaL] A. Katok and M. Lemańczyk, Some new cases of realization of spectral multiplicity function for ergodic transformations, Fund. Math. 206 (2009), 185-215.

[KaS] A. B. Katok and A. M. Stepin, Metric properties of homeomorphisms that preserve measure, Uspekhi Mat. Nauk 25 (1970), no. 2, 193-220.

[Ko] R. A. Konev, On the collection $\left\{2, \ldots, 2^{n}\right\}$ of spectral multiplicities for an ergodic $\mathbb{Z}^{2}$-action, in preparation. 
[KL] J. Kwiatkowski (jr) and M. Lemańczyk, On the multiplicity function of ergodic group extensions. II, Studia Math. 116 (1995), 207-215.

[Os] V. I. Oseledec, On the spectrum of ergodic automorphisms, Soviet Math. Dokl. 168 (1966), 776-779.

[R1] E. A. Robinson, Ergodic measure-preserving transformations with arbitrary finite spectral multiplicities, Invent. Math. 72 (1983), 299-314.

[R2] - Transformations with highly nonhomogeneous spectrum of finite multiplicity, Israel J. Math. 56 (1986), 75-88.

[Ry1] V. V. Ryzhikov, Transformations having homogeneous spectra, J. Dynam. Control Systems 5 (1999), 145-148.

[Ry2] - Homogeneous spectrum, disjointness of convolutions, and mixing properties of dynamical systems, Selected Russian Math. 1 (1999), 13-24.

[Ry3] - Weak limits of powers, the simple spectrum of symmetric products and mixing constructions of rank 1, Mat. Sb. 198 (2007), 137-159 (in Russian).

[Ry4] - Spectral multiplicities and asymptotic operator properties of actions with invariant measure, ibid., to appear.

Alexandre I. Danilenko

Institute for Low Temperature Physics Engineering

National Academy of Sciences of Ukraine

47 Lenin Ave.

Kharkov, 61164, Ukraine

E-mail: alexandre.danilenko@gmail.com 